Image Processing, Geoinformatics and Information Security

\title{
HYPERSPECTRAL IMAGE COMPRESSION FOR TRANSMISSION OVER COMMUNICATION CHANNEL
}

\author{
N.I. Glumov, M.V. Gashnikov \\ Samara National Research University, Samara, Russia
}

\begin{abstract}
In this paper, we describe a modification of the previously developed on-board image processing method applied to hyperspectral images. Algorithms on which the method is based were finalized and parametrically adjusted. Computational experiments consider formation and storage specifics for hyperspectral images. It has been shown that the proposed method based on HGIcompression can be recommended for implementation in on-board processing systems and transmission over communication channels.
\end{abstract}

Keywords: hyperspectral images, compression data, hierarchical grid interpolation method, on-board processing.

Citation: Glumov NI, Gashnikov MV. Hyperspectral image compression for transmission over communication channel. CEUR Workshop Proceedings, 2016; 1638: 334-339. DOI: 10.18287/1613-0073-2016-1638-334-339

Specifics of real-time image processing systems, particularly operational remote sensing systems [1-2], primarily depend on the following characteristics:

- high speed of input image formation;

- digital constant bandwidth communication channel availability;

- the possibility of communication channel random failures;

- - tight restrictions on hardware specifications (weight, power consumption and etc.);

- a wide range of problems, which are not predetermined and which can be solved by using the resulting images.

These characteristics determine the following requirements for on-board image processing methods:

- high compression ratio [3] (the ratio between the original and the compressed amount of information);

- strict control of quality [4], estimated by the maximum or the mean square reconstruction error;

- low computational and structural complexity; 
- constant speed of the compressed data output stream generation;

- high noise immunity of output data.

Compression methods based on discrete transformations (cosine transform, wavelet transform) do not meet the above-mentioned requirements, primarily the complexity and quality control capabilities. Generally, more simple compression schemes based on the differential coding are used in real-time systems. However, these methods have low compression ratio.

Compression method based on the hierarchical grid interpolation (HGI-method) [5] meets all the compression ratio, quality control and algorithm complexity requirements. However, the method needs the further improvement to provide not only data compression problem solution, but also constant speed of the compressed data output stream generation and high noise immunity of output data.

The general scheme of the on-board image processing method is given in Figure 1 [5]. Three separate blocks of the scheme describe the solution for the problems of compression, output data speed stabilization and protection of encoded information against communication channel failures.

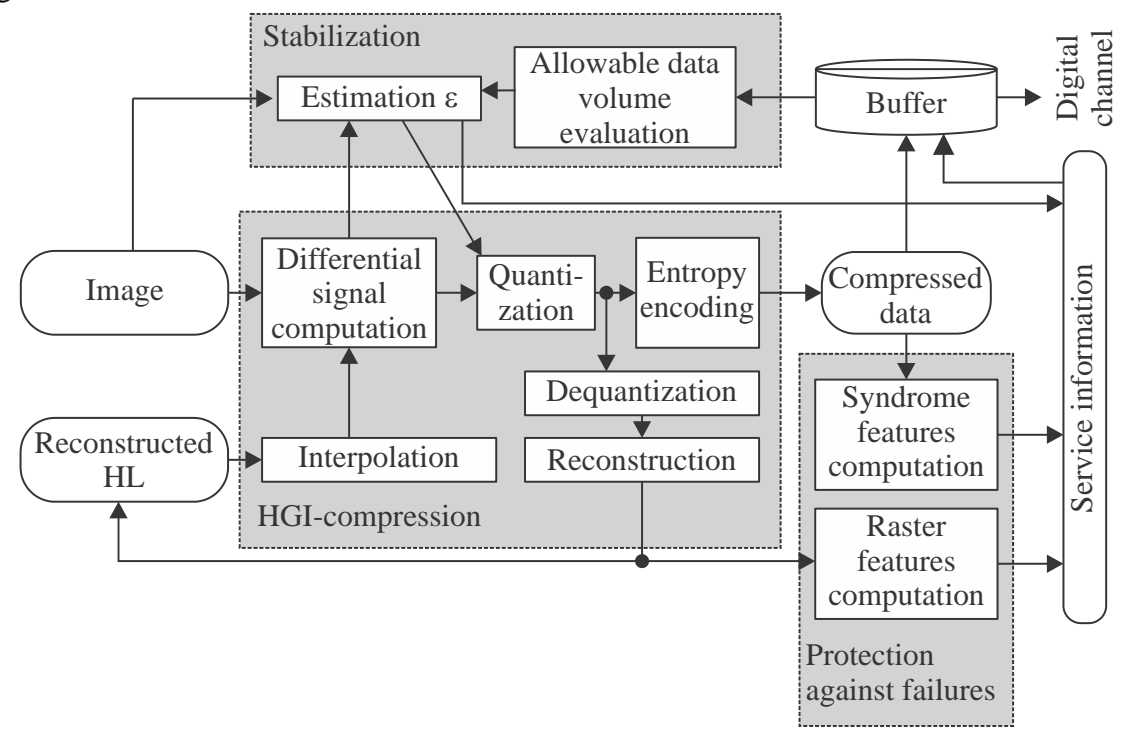

Fig. 1. The general scheme of the on-board image processing

The authors propose using this scheme for monochrome image generation and transmission over communication channels.

Hyperspectral image generation [6-8] while transmitting over communication channels, in particular on board the aircraft, usually has its own specifics, depending on the design of the sensors used for the hyperspectral data registration. The sequentially supplied two-dimensional images will be the input to the compression procedure and the totality of these images will represent, in essence, resulting hyperspectral data. The specificity lies in the fact that unlike the usual case of work with hyperspectral images these two-dimensional images do not comprise spectral components. The first 
two-dimensional image contains the first lines of all spectral components; the second two-dimensional image contains all second lines and etc. In other words, the "rotated" "hyperspectral cube" serves as the input to a compression method. Such feature of hyperspectral cube orientation does not entail any problems in implementing the compression method, because if there are several hundred components, the twodimensional images, consisting of the original hyperspectral data corresponding lines, may be considered as the spectral components. That is, instead of hyperspectral image comprising $\mathrm{S}$ components of size $V \times H$ pixels, the result of a compression method will be the hyperspectral image comprising V components of size $S \times H$ pixels.

Figures 2-3 show the examples of the individual components of the hyperspectral cube, based on satellite image fragment made by spectrometer AVIRIS [9].
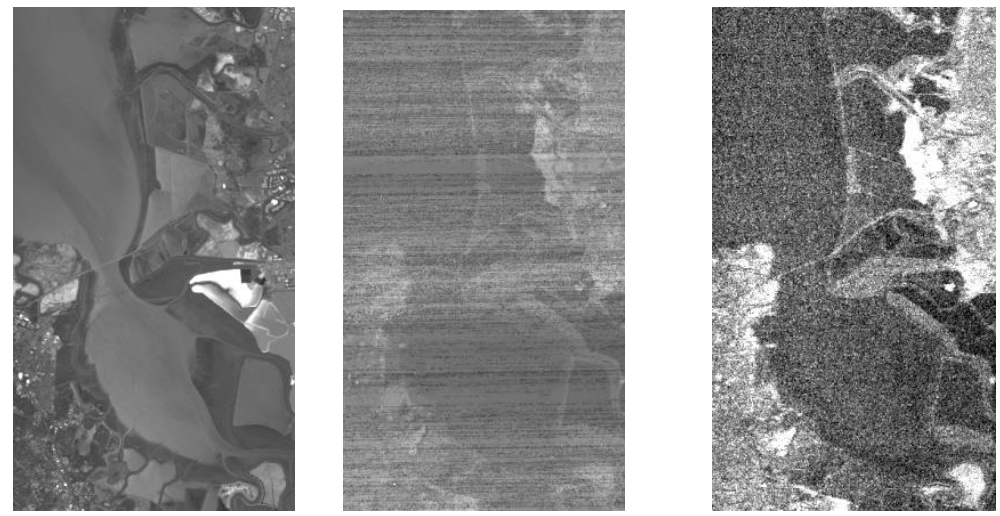

Fig. 2. Components № 24, 107, 159 of the "mf16a" image

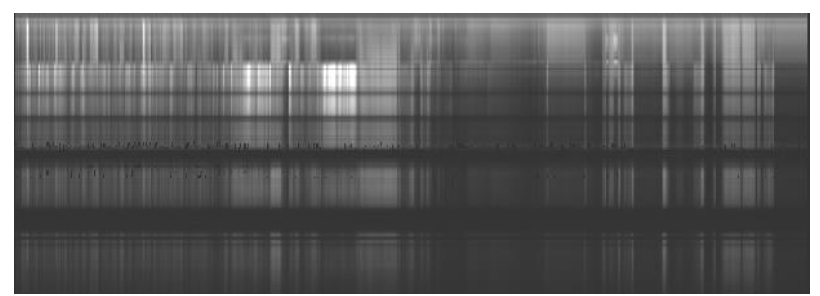

Fig. 3. Component № 1068 of the "detailed" "mf16a" image

Compression algorithms based on HGI-method, proposed in [10-13], consider strong correlation between hyperspectral image components and based on "sliding component approximation", "non-overlapping portions of components" and "shared support components". The most effective (by the criterion of the compression ratio at a fixed data recovery error) was the algorithm based on "shared support components" and it can be recommended for using HGI-method in case of hyperspectral image database storage.

In this paper, statistical characteristics of the "detailed" hyperspectral images were analyzed. The analysis has shown that they are much less "convenient" for compression than the original "non-detailed". "Detailed" images have a greater dispersion, and dispersion is high for all components; the dispersion of «non-detailed» images has 
been decreasing for many components, which led to the compression ratio increase, and therefore the compression ratio was significantly deteriorated. On the other hand, "detailed" hyperspectral image correlation between components is significantly lower than correlation between components of the original hyperspectral image.

These features can be ignored for implementation of the HGI-method basic algorithms: processing of the image hierarchical levels, quantization, and entropy encoding. However, they significantly influence the choice of the algorithm optimal parameters, and eventually affect the efficiency of the compression method.

Figure 4 shows the results of computational experiments for the original and "detailed" hyperspectral images as average correlation between the compression ratio and the mean square/maximum reconstruction error over the set of images AVIRIS for the above-mentioned algorithms and also for the algorithm of hyperspectral component independent compression.
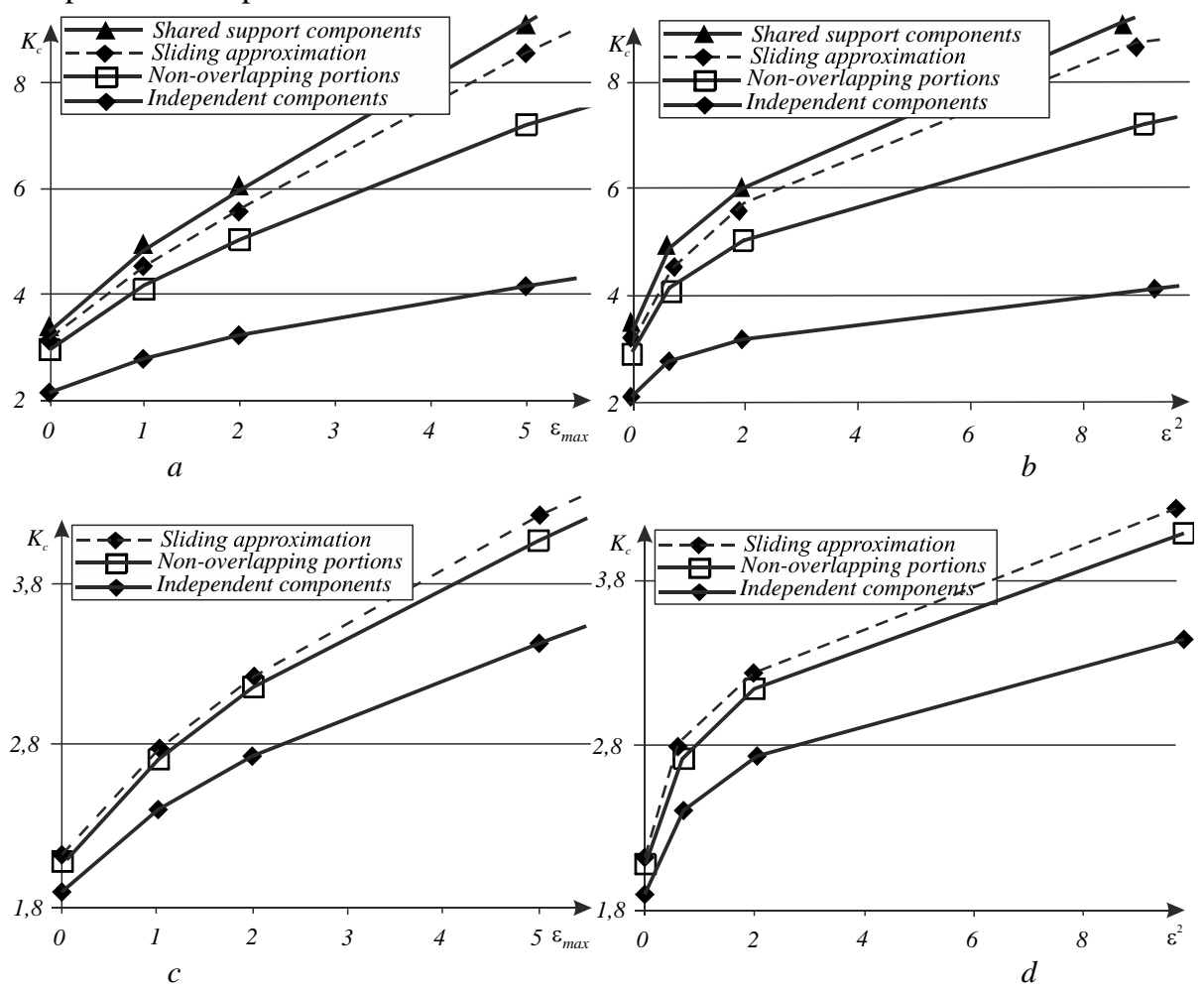

Fig. 4. The average compression ratio for the five images depending on the maximum $\varepsilon_{\max }$

and $\varepsilon^{2}$ the mean square errors: (a)-(b) for original hyperspectral images,

(c)-(d) - for "detailed" hyperspectral images

It should be noted that when selecting hyperspectral image compression algorithm for transmission over communication channels we should consider the fact that random access memory of such systems is severely limited and simultaneous spectral compo- 
nent storage, required in the implementation of approximation compression algorithms, is undesirable. Thus, for the systems of hyperspectral image generation and transmission over communication channels the selection of the algorithm based on independent HGI-compression of components seems to be the most suitable because of its sufficiently high compression ratio.

The specifics of using the HGI-method for the remote sensing data transmission lies in the fact, that the controlled error, we use in this method, entails the variable speed of compressed data stream generation (compression ratio is unstable over time). This drawback limits the method application in real-time image generation and processing systems with fixed-bandwidth communication channels, including remote sensing data transmission systems. Generally, such obstacle can be eliminated by buffering the output data, that is, the use of the buffer memory (hereinafter referred to as buffer). When buffering, portions of data are compressed, and for each portion control parameters of the compression method are selected so as to prevent buffer overflow. For the HGI-method the maximum error serves as a control parameter.

An adaptive stabilization algorithm, proposed in [5,14], is adjustable to the features of each compressed portion of data. To determine the maximum error $\varepsilon_{\max }(t)$ for another portion number $t$ the statistical characteristics of portion (dispersion $D(t)$ and correlation coefficient $\rho(t))$ and the permissible degree of compression estimation $\hat{B}(t)$ (bit/count), which provides buffering of the compressed portion of data, are used:

$$
\varepsilon_{\max }(t)=f(D(t), \rho(t), \hat{B}(t)) .
$$

In this paper, for a large set of different portions of real hyperspectral images the value tables of $\varepsilon_{\max }(t), D(t), \rho(t)$ and the corresponding values $B(t)$, obtained as a result of compression, were built by the computational experiment. Using the data from the table, we approximated the correlation and calculated parameters of the function, through which the desired $\varepsilon_{\max }(t)$ can be calculated. We propose to use a linear function

$$
\varepsilon_{\max }(t)=a_{0}+a_{1} D(t)^{k_{1}}+a_{2} \rho(t)^{k_{2}}+a_{3} \hat{B}(t)^{k_{3}}
$$

The parameters $a_{i}, k_{i}$ of the function were determined during the computational experiment, and the results showed, that, in $95.1 \%$ of cases, the deviation of resulting compression ratio from the fixed one was $|\Delta B| \leq 0.2 \mathrm{bit} /$ pixel and confirmed the possibility of implementing the algorithm of the speed stabilization of the compressed hyperspectral images generation in conditions of limited capacity of the buffer memory and communication channel capacity.

The last block of the proposed on-board hyperspectral image processing scheme (see. Fig.1) is the use of the noise immunity improvement algorithm [5,15]. According to experimental studies, the specifics of hyperspectral images do not impose new re- 
strictions on the developed algorithm for monochrome images, and it can be recommended for compressed hyperspectral images processing.

\section{Acknowledgements}

This work was financially supported by the Russian Scientific Foundation (RSF), grant no. 14-31-00014 "Establishment of a Laboratory of Advanced Technology for Earth Remote Sensing".

\section{References}

1. Borengasser M, Hungate W, Watkins R. Hyperspectral Remote Sensing. Principles and Applications. CRC Press, 2004; 128 p.

2. Chang C. Hyperspectral Data Processing: Algorithm Design and Analysis. Wiley Press, 2013; 1164 p.

3. Salomon D. Data Compression. The Complete Reference. Springer-Verlag, 4ed, 2007; 1118 p.

4. Woods E, Gonzalez R. Digital Image Processing. Prentice Hall, 3ed, 2007; 976 p.

5. Gashnikov MV, Glumov NI, Sergeyev VV. A hierarchical compression method for space images. Automation and Remote Control, 2010; 7(3): 501-513.

6. Chang C. Hyperspectral data exploitation: theory and applications. WileyInterscience, 2007; 440 p.

7. Benz U, Hofmann P, Willhauck G, Lingenfelder I, Heynen M. Multi-resolution, object-oriented fuzzy analysis of remote sensing data for GIS-ready information. ISPRS Journal of Photogrammetry and Remote Sensing, 2004; 58(3): 239-258.

8. Chang C, Chiang S. Anomaly detection and classification for hyperspectral imagery. IEEE Transactions on Geoscience and Remote Sensing, 2002; 40(6): 1314-1325.

9. Ordering Free AVIRIS Standard Data Products. Jet Propulsion Laboratory. URL: http://aviris.jpl.na-sa.gov/data/free_data.html

10. Gashnikov MV, Glumov NI. Hierarchical GRID Interpolation under Hyperspectral Images Compression. Computer Optics, 2014; 38(1): 87-93. [in Russian]

11. Gashnikov MV, Glumov NI. Hierarchical GRID Interpolation under Hyperspectral Images Compression. Optical Memory and Neural Networks (Information Optics), 2014; 23(4): 246-253.

12. Gashnikov MV, Glumov NI. Hierarchical compression in the problem of hyperspectral image storage. Computer Optics, 2014; 38(3): 482-488. [in Russian]

13. Gashnikov MV. Parameterization of nonlinear Greham predictor for digital image compression. Computer Optics, 2016; 40(2): 225-231. [in Russian]

14. Gashnikov MV, Glumov NI, Sergeyev VV. Stabilization of the Compressed Data Formation Rate in Hierarchical Image Compression. Pattern Recognition and Image Analysis, 2007; 17(1): 79-81.

15. Glumov NI. Improving Noise Immunity of Transmission of Compressed Digital Images Pattern Recognition and Image Analysis, 2003; 13(2): 273-276. 